\title{
Preoperative perihilar cholangiocarcinoma assessment using virtual endoscopic imaging magnetic resonance cholangioscopy
}

\section{(ㄷ)(우우}

\author{
Authors \\ Akinori Maruta ${ }^{3}$, Keisuke Iwata ${ }^{3}$, Eiichi Tomita', Masahito Shimizu² \\ Institutions \\ 1 Department of Gastroenterology, Gifu Municipal \\ Hospital, Gifu, Japan \\ 2 First Department of Internal Medicine, Gifu University \\ Hospital, Gifu, Japan \\ 3 Department of Gastroenterology, Gifu Prefecture \\ General Medical Center, Gifu, Japan
}

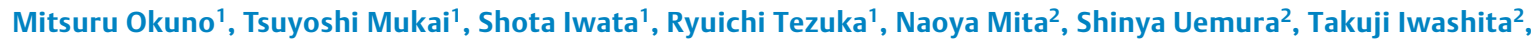

submitted 2.12 .2020

accepted after revision 15.2.2021

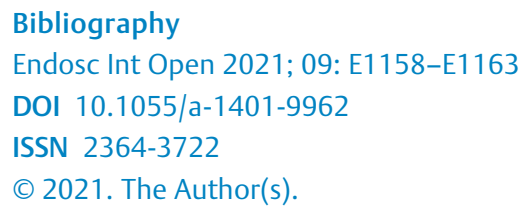

\author{
\# Supplementary material is available under \\ https://doi.org/10.1055/a-1401-9962
}

\section{ABSTRACT}

Background and study aims Preoperative assessment of the superficial ductal spread (SDS) of perihilar cholangiocarcinoma (PCCA) is important for determining its resectability. A virtual endoscopic imaging method, magnetic resonance cholangioscopy (MRCS), wherein a three-dimensional image is created from magnetic resonance imaging (MRI) data, can evaluate all aspects of arbitrary bile ducts.

Patients and methods Overall, 15 patients with PCCA who underwent preoperative MRI were enrolled. All patients underwent surgical treatment.

Results MRCS could be performed based on preoperative MRI data in all patients. MRCS could not be used in one patient due to debris in the obstructed bile duct. The remaining 14 patients ( $93 \%$ ) were diagnosed with obstructed bile duct or irregular surface of the bile duct, signifying tumor invasion. The accuracy rate of diagnosing the SDS was $93 \%$ $(14 / 15)$. Seven patients underwent preoperative peroral cholangioscopy; the scope could not pass through the tumor in three patients. In these three patients, MRCS was able to be use dtoevaluate the obstructed side of the bile duct and faciliated accurate diagnosis of SDS.

Conclusions MRCS can be used to noninvasively evaluate the bile duct in all directions. This novel method makes it easy to create virtual images and can be useful for diagnosing the preoperative SDS of PCCA.

\section{Introduction}

Complete resection is a significant factor influencing long-term survival in patients with cholangiocarcinoma (CCA). A negative margin is required for complete resection; therefore, an accurate preoperative diagnosis of tumor extension is important. In patients with perihilar CCA (PCCA), preoperative assessment of the superficial ductal spread (SDS) is important for deciding resectability; however, this is sometimes difficult due to the anatomical complexity of the bile ducts. Magnetic resonance cholangiopancreatography (MRCP) can provide a clear threedimensional ( $3 D$ ) image of the anatomy of the bile duct without being invasive [1]. The bile duct outline can be evaluated using MRCP imaging; however, the SDS of PCCA is sometimes difficult to evaluate with MRCP as well [2]. As a solution to this, virtual endoscopic imaging has been developed; using two-dimensional (2D) images obtained with MRCP, $3 \mathrm{D}$ images can be created. For this, the $3 \mathrm{D}$ image software (SYNAPSE VIN- 


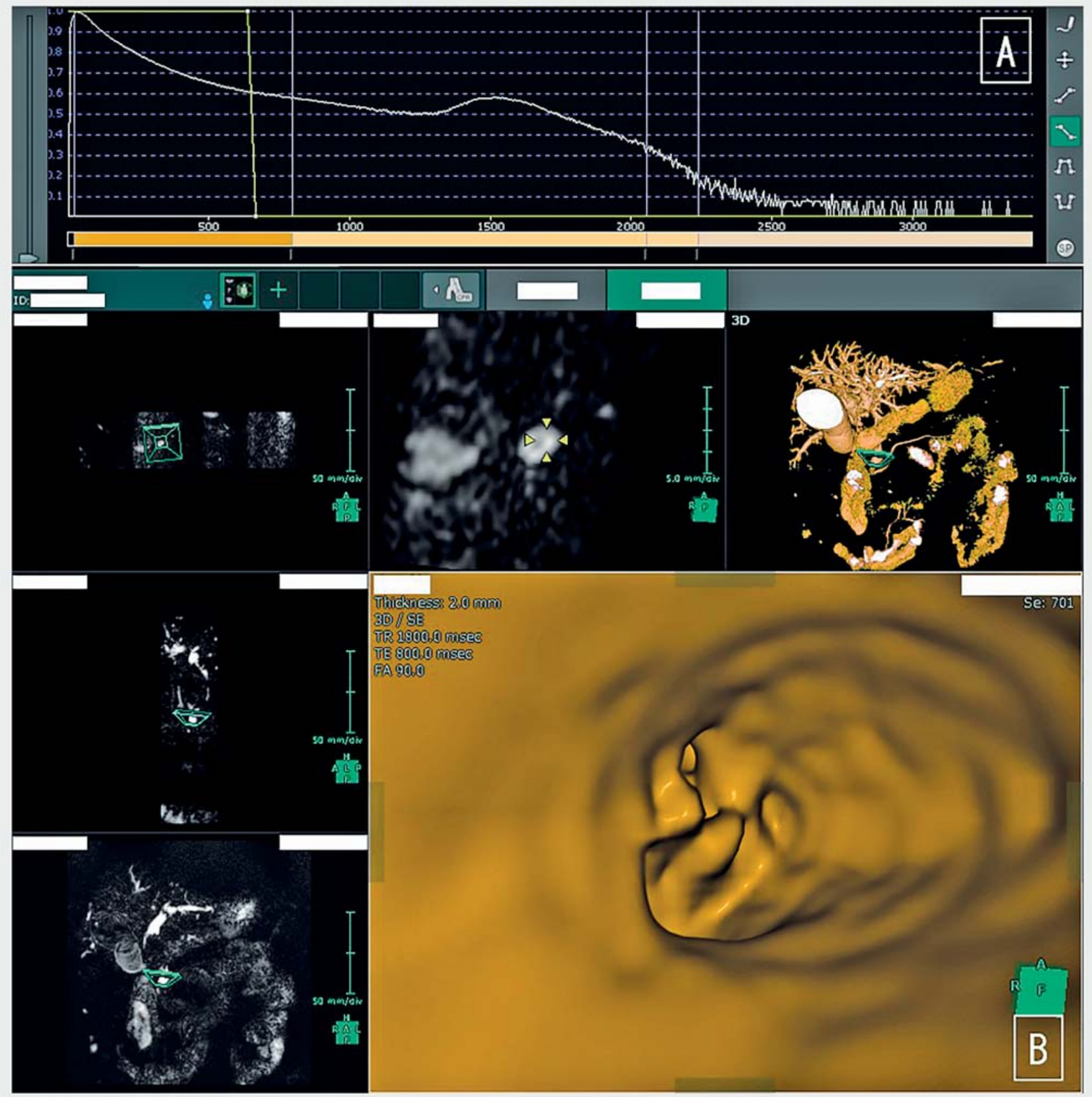

- Fig. 1 A The opacity of the MRCP data was set to less than 1000 for tracing the outline of the bile duct. B The created MRCS image was evaluated using virtual endoscopic view mode of SYNAPSE VINCENT system (Fujifilm Medical, Tokyo, Japan). MRCP, magnetic resonance cholangiopancreatography; MRCS, magnetic resonance cholangioscopy.

CENT system [Fujifilm Medical, Tokyo, Japan]) is primarily used. We named this technique magnetic resonance cholangioscopy (MRCS). MRCS makes arbitrary visualization of the bile duct in all directions possible through the use of $3 \mathrm{D}$ images.

\section{Patients and methods}

\section{Study design}

This retrospective, observational study aimed to evaluate the accuracy of MRCS for the preoperative SDS of PCCA. This study also evaluated whether MRCS, which is a non-invasive test, has diagnostic capabilities different from those of the existing diagnostic methods for ERC and POCS. We enrolled the patients who met the enrollment criteria from clinical databases. The 
study protocol was approved by the institutional review board of Gifu Municipal Hospital, Gifu University Hospital, and Gifu Prefectural General Medical Center, and registered with the University Hospital Medical Information Network Clinical Trial Registry (UMIN000040708).

\section{Patients}

Patients diagnosed with resectable PCCA based on pathological and typical radiologic findings were included and evaluated using MRCP before the endoscopic retrograde cholangiography (ERC) procedure and surgical treatment between May 2005 and January 2020. The exclusion criteria were as follows: those aged $<20$ years, those who underwent magnetic resonance imaging (MRI) after biliary drainage, and those unable to provide informed consent. Fifteen consecutive PCCA patients were enrolled. Peroral cholangioscopy (POCS) was performed in seven of 15 patients before surgical treatment. The final diagnosis of the SDS of PCCA was confirmed based on the pathological findings of the surgical specimens. Written informed consent was obtained from all patients. The study was conducted in accordance with the guidelines provided in the Declaration of Helsinki.

\section{Magnetic resonance cholangioscopy}

Patients underwent 1.5T MRI scans (Intera 1.5T or Achieva 1.5T; Philips, Netherlands). MRI data were transferred to a SYNAPSE VINCENT workstation. MRCS was created using the software in the $3 \mathrm{D}$ view mode by an endoscopist. The opacity of MRCP data was set to less than 1000 to enable tracing the bile duct outline ( $\triangleright$ Fig. 1A). The created 3D image was evaluated using the virtual endoscopic view mode available in the software ( $\triangleright$ Fig. 1B). The MRCS image was evaluated by two expert therapeutic endoscopists who had a combined experience of performing over 50 POCS and 2000 ERC procedures. They did not determine the accurate location of the SDS based on pathological findings.

\section{ERC procedure}

ERC was performed using a standard duodenoscope (TJF-200, TJF-240, or TJF-260V; Olympus Medical System, Tokyo, Japan). Seven patients were evaluated using a video cholangioscope (CHF-B260; Olympus Medical System, Tokyo, Japan, or SpyGlass DS; Boston Scientific, Massachusetts, United States) after endoscopic sphincterotomy. To observe the biliary mucosa clearly during POCS, the bile duct was irrigated with sterile saline through the working channel. White light imaging with/ without narrow-band imaging, which was used when the CHFB260 was selected, was utilized for evaluating the bile duct. The SDS of malignancy was defined as the presence of irregular surface, easy oozing from the bile duct mucosa, irregularly dilated vessels, and luminal narrowing.

\section{Study outcomes}

The outcome of this study was determining the accuracy of MRCS in the preoperative SDS diagnosis of PCCA. The SDS was evaluated on both the distal bile duct side (papillary side) and the hepatic duct using antegrade and retrograde virtual endoscopic views ( $\triangleright$ Fig. 2 ). The other visual findings of SDS accura-
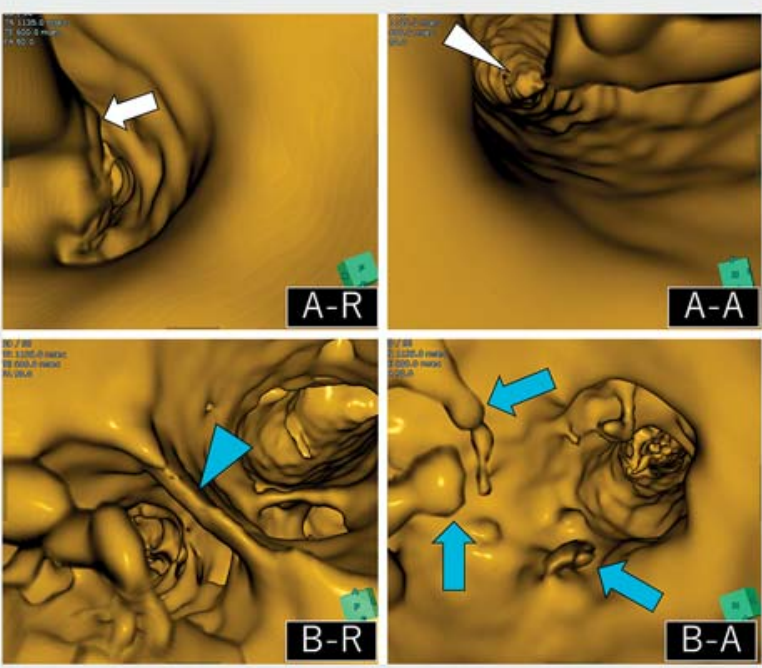

- Fig. 2 MRCS can evaluate the superficial ductal spread of the distal and hepatic bile duct. A-R The distal biliary stricture (arrow) can be detected under the retrograde view from distal bile duct. A-A The main papilla (arrowhead) can be detected from the distal end of tumor, with the smooth common bile duct wall under the antegrade view. B-R The sharp corner of the hepatic bile duct branching without tumor invasion (blue arrowhead) can be evaluated under the retrograde view from the hepatic bile duct. B-A The papillary tumor that is invasive to the opposite side of the hilar bile duct branch (blue arrowhead) can be evaluated under the antegrade view. MRCS: magnetic resonance cholangioscopy.

cy, including ERC and POCS, were also evaluated. Diagnostic accuracy was determined by comparing the results of visual findings to the pathological findings of surgical specimens.

\section{Results}

\section{Patient characteristics}

Fifteen patients (14 men; median age, 71 years [range, 46-85 years] were enrolled. The patients' basic characteristics are summarized in $>$ Table 1 . The Bismuth classifications of MRCP and the initial ERC findings were type I in five patients, type II in six, type IIla in two, and type IV in two patients. Seven patients $(47 \%)$ underwent POCS for preoperative assessment. The operative procedures performed were hepatectomy+bile duct resection in 10 patients, pancreatoduodenectomy in three, and hepatopancreatoduodenectomy in two.

\section{Evaluation of the superficial ductal spread with MRCS, ERC, and POCS}

MRCS was successfully created and evaluated in all patients. The main tumor, the branches of the hepatic bile duct (hepatic side), and the intrapancreatic common bile duct (papillary side) were evaluated. The obstructed branched bile duct and the common bile duct, or the irregular surface of the bile duct, were detected as tumor invasion using retrograde and antegrade view ( $>$ Fig. 2 ). The intact bile duct (benign) could be identified as a smooth bile duct wall and a sharp corner of the 
branched bile duct in the hepatic duct ( $\mathbf{F i g}$. 2B-R). The intact intrapancreatic common bile duct could also be identified with the smooth bile duct wall ( $\nabla$ Fig. 2A-A). The presence of a distal biliary stricture ( $\mathbf{F i g . 2 A - R}$ ) and the irregular surface of the bile duct wall ( $>$ Fig. 2B-A) were evaluated as malignant SDS. Debris was present in the obstructed bile duct in one case; as the debris appeared as suspended matter, this case was considered overdiagnosed. The accuracy rate of the SDS diagnosis in MRCS was $93 \%(14 / 15)$ in the hepatic side and $100 \%(15 / 15)$ in the papillary side ( $\triangleright$ Table 2 ).

The accuracy rate of the SDS diagnosis in ERC was $87 \%$ $(13 / 15)$ in the hepatic side and $100 \%(15 / 15)$ in the papillary side ( $\triangleright$ Table 2 ). Because the contrast medium could not pool accurately in the obstructed bile duct due to the bile duct bending in the hepatic side, two patients were considered as cases of SDS overdiagnosis. However, these two cases could be accurately diagnosed using MRCS. The accuracy rate of the SDS diagnosis in ERC with MRCS was $100 \%(15 / 15)$ in the hepatic side ( $>$ Table 3 ).

POCS was performed in seven cases. POCS findings of the SDS in the bile duct mucosa included an irregular fine granular pattern and a fish-egg-like pattern with small red nodules in the center. The scope did not pass through the tumor obstruction in three patients during POCS; the SDS at the hepatic side could, therefore, not be diagnosed in them. When these three cases were excluded, the accuracy rate of the hepatic side SDS diagnosis in POCS was $100 \%$ (3/3). The intrapancreatic bile duct could be evaluated in all seven POCS cases. The accuracy rate of the SDS diagnosis was $100 \%$ (7/7) in the papillary side $(\triangleright$ Table 2). MRCS could evaluate the SDS of the hepatic side
- Table 1 Overall characteristics of 15 patients who were evaluated using MRCP for perihilar cholangiocarcinoma.

Age (years), mean (IQR)

$71(46-85)$

Female sex, $\mathrm{n}(\%)$

$1(7 \%)$

Bismuth classification

- I/II/IIla/IV

- No. of patients who underwent POCS, n (\%) $7(47 \%)$

- No. patients in whom POCS could pass across a $4 / 7(57 \%)$ biliary obstruction, $\mathrm{n}(\%)$

Type of operation, $\mathrm{n}(\%)$

- Hepatectomy + bile duct resection $10(67 \%)$

- Pancreatoduodenectomy $3(20 \%)$

- Hepatopancreatoduodenectomy $2(13 \%)$

$\mathrm{IQR}$, interquartile range; MRCP, magnetic resonance cholangiopancreatography; POCS, peroral cholangioscopy.

correctly in these three cases. MRCS enabled definite evaluation of the sharp corner of the hepatic bile duct branch without tumor invasion in the retrograde view, and the obstructed bile duct wall was observed from the opposite side of the hilar bile duct branching using the antegrade view ( $\triangleright$ Fig.3). The accuracy rate of the SDS diagnosis in POCS with MRCS was $100 \%(7 / 7)$ in the hepatic side ( $\triangleright$ Table 3 ). With the addition of MRCS to ERC or POCS, SDS was diagnosed in all cases.

- Table2 Diagnostic accuracy values of MRCS, ERC, and POCS for the superficial ductal spread of perihilar cholangiocarcinoma.

\begin{tabular}{|c|c|c|c|c|c|c|}
\hline & MRCS & & ERC & & POCS & \\
\hline & $\begin{array}{l}\text { Hepatic side } \\
(n=15)\end{array}$ & $\begin{array}{l}\text { Papillary side } \\
(n=15)\end{array}$ & $\begin{array}{l}\text { Hepatic side } \\
(n=15)\end{array}$ & $\begin{array}{l}\text { Papillary side } \\
(n=15)\end{array}$ & $\begin{array}{l}\text { Hepatic side } \\
(n=4)^{*}\end{array}$ & $\begin{array}{l}\text { Papillary side } \\
(n=7)\end{array}$ \\
\hline Accuracy & $93 \%(14 / 15)$ & $100 \%(15 / 15)$ & $87 \%(13 / 15)$ & $100 \%(15 / 15)$ & $100 \%(4 / 4)$ & $100 \%(7 / 7)$ \\
\hline Overdiagnosis & $7 \%(1 / 15)$ & $0 \%$ & $13 \%(2 / 15)$ & $0 \%$ & $0 \%$ & $0 \%$ \\
\hline Underdiagnosis & $0 \%$ & $0 \%$ & $0 \%$ & $0 \%$ & $0 \%$ & $0 \%$ \\
\hline
\end{tabular}

- Table 3 Hepatic side bile duct diagnostic accuracy values of ERC or POCS with/without MRCSfor the superficial ductal spread of perihilar cholangiocarcinoma.

\begin{tabular}{|l|c|c|c|}
\hline & Accurate diagnosis & Over diagnosis & Under diagnosis \\
\hline ERC alone & $87 \%(13 / 15)$ & $13 \%(2 / 15)$ & $0 \%$ \\
\hline ERC with MRCS & $100 \%(15 / 15)$ & $0 \%$ & Non-evaluation due to tumor obstruction \\
\hline & Accurate diagnosis & $43 \%(3 / 7)$ \\
\hline POCS alone & $57 \%(4 / 7)$ & $0 \%$ & $0 \%$ \\
\hline POCS with MRCS & $100 \%(7 / 7)$ & \\
\hline ERC, endoscopic retrograde cholangiography; MRCS, magnetic resonance cholangioscopy; POCS: peroral cholangioscopy.
\end{tabular}



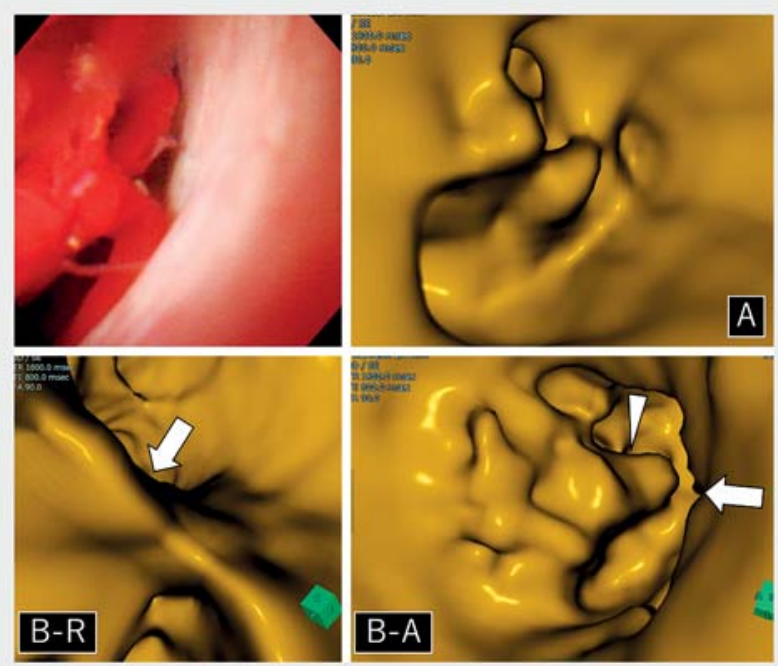

- Fig. 3 A POCS (distal bile duct retrograde view) cannot pass the tumor obstruction. MRCS shows the obstructed common bile duct by the tumor in the retrograde view. B-R MRCS evaluating the hepatic side of the tumor, which was evaluated as the sharp corner of the distal branched bile duct in the retrograde view (arrow). B-A The obstructed bile duct wall can be observed from the opposite side of the hilar bile duct branch under the antegrade view (arrowhead). MRCS, magnetic resonance cholangioscopy; POCS, peroral cholangioscopy.

\section{Discussion}

Preoperative SDS of PCCA is important information for determining the surgical resectability of the condition. MRCP is used routinely for evaluating biliary strictures. The accuracy rate of MRCP in diagnosing biliary strictures was reported to be $82 \%$ to $98 \%$ [3-7]. MRCP provides results similar to those provided by ERC and is also useful for situations where ERC is difficult, such as those involving a complex hilar stricture $[5,8]$. MRCP evaluates the outline of the bile duct; therefore, smaller tumors may often be obscured by overlapping bile ducts and thus missed.

MRCS was created from the 2D images obtained using MRCP, which only shows the bile duct lumen. The bile duct lumen displays as white in MRCP. The whiteness is stronger in the center of the bile duct than around its surface; hence, just setting the opacity to less than 1000 in the software when assessing MRCP data can enable clear tracking of only the outline of the bile duct ( $\triangleright$ Fig. 1a).

ERC is the most common imaging test for diagnosing the SDS of PCCD. However, it is difficult to evaluate the presence of mass lesions around the PCCA because the contrast medium is sometimes difficult to pool accurately around the tumor [8]. In our study, the accuracy rate of ERC for bile duct diagnosis was $87 \%(13 / 15)$ in the hepatic side, which includes the two overdiagnosis cases that were caused by difficulty in pooling the contrast medium around the tumor. These overdiagnosis cases could be diagnosed accurately in MRCS. Because the obstructed side bile duct, including the area around the tumor, is filled by bile, MRCS could detect and evaluate the surface of the bile duct around the tumor. This advantage can improve the accuracy of diagnosis.

POCS is a useful procedure for diagnosing SDS because the video endoscope can provide high-resolution images $[9,10]$. - Fig. 4 shows the comparison of MRCS and POCS images from our study. MRCS can evaluate the surface structure, which is similar to the POCS image. However, MRCS cannot evaluate the vascular structure; this is a disadvantage of MRCS.

The major advantage of MRCS is that it enables non-invasive arbitrary evaluation of the bile duct in all directions. In previous reports on POCS, the scope could not be passed through the tumor obstruction in some cases, which reduced the diagnostic accuracy of POCS [9]. Bends in the bile duct also make it difficult to insert the scope during POCS. In our study, there were 3 cases wherein the hepatic side could not be evaluated due to tumor obstruction. MRCS enabled accurate evaluation in all these three cases. Therefore, MRCS may be an alternative method and good indication for evaluation when POCS cannot be performed in case of tumor obstruction and bile duct bending. Moreover, substituting POCS with MRCS has the potential to reduce the cost of POCS-related adverse events and devices.

Our study has some limitations. This study was retrospective and had a small number of cholangiocarcinoma cases that included only four intrahepatic bile duct invasion cases (Bismuth type III or IV). There were also only seven POCS cases, and POCS could not be performed in the remaining eight cases due to a narrow distal bile duct and/or long (>30 mm) malignant biliary stricture. There was no difference in the bile duct lumen evaluation for the SDS diagnosis between MRCS and POCS in this study; however, further verification of the effectiveness of MRCS images confirmed using prospective studies with a greater number of patients, focusing on image comparison between POCS and MRCS, is necessary. Second, the cases of obstructed bile duct filled by debris could not be evaluated by MRCS; this has a significant potential to be misidentified as the tumor. Therefore, when ERC indicates obstructed bile duct with debris, it can be difficult to evaluate the SDS of PCCA using MRCS.

\section{Conclusions}

In conclusion, MRCS is considered as a new method for the accurate diagnosis of the SDS of PCCA. This novel method can be easily created from MRI data and can noninvasively evaluate arbitrary bile ducts.

\section{Acknowledgents}

The authors thank all the patients and support staff who participated in this study. 

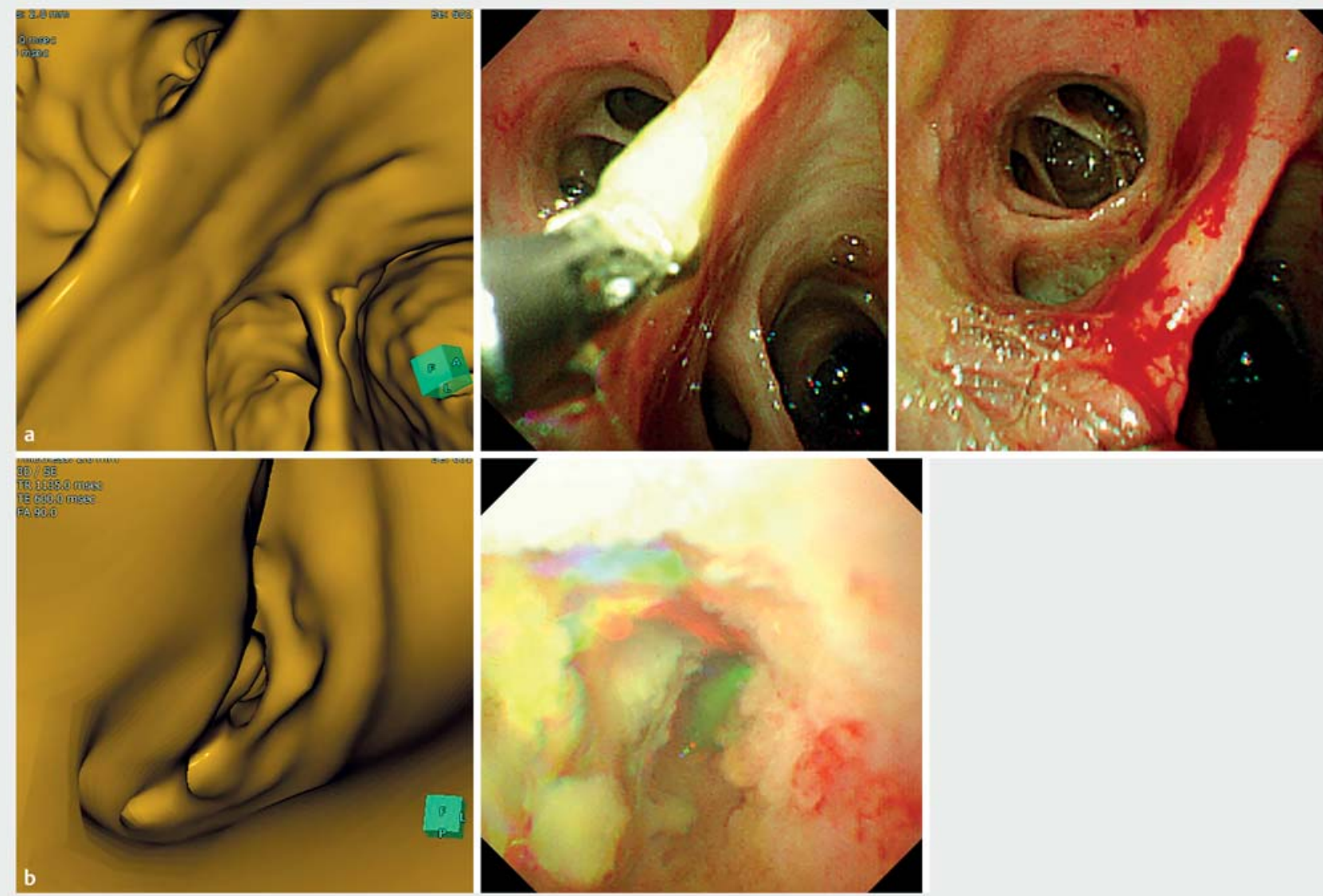

Fig. 4 Comparison of POCS and MRCS images. a The benign bile duct wall. The smooth bile duct wall and the sharp corner of the branched bile duct were detected in MRCS. $\mathbf{b}$ The malignant bile duct wall. The bulged and irregular surface of the bile duct wall was detected in MRCS. MRCS can evaluate the surface structure similar to the POCS image. However, MRCS cannot evaluate the vascular structure. MRCS, magnetic resonance cholangioscopy; POCS, peroral cholangioscopy.

\section{Competing interests}

The authors declare that they have no conflict of interest.

\section{References}

[1] Hintze RE, Abou-Rebyeh H, Adler A et al. Magnetic resonance cholangiopancreatography-guided unilateral endoscopic stent placement for Klatskin tumors. Gastrointest Endosc 2001; 53: 40-46

[2] Lee SS, Kim MH, Lee SK et al. MR cholangiography versus cholangioscopy for evaluation of longitudinal extension of hilar cholangiocarcinoma. Gastrointest Endosc 2002; 56: 25-32

[3] Materne R, Van Beers BE, Gigot JF et al. Extrahepatic biliary obstruction: magnetic resonance imaging compared with endoscopic ultrasonography. Endoscopy 2000; 32: 3-9

[4] Blechacz B, Gores G]. Cholangiocarcinoma: advances in pathogenesis, diagnosis, and treatment. Hepatology 2008; 48: 308-321
[5] Adamek HE, Albert J, Weitz $\mathrm{M}$ et al. A prospective evaluation of magnetic resonance cholangiopancreatography in patients with suspected bile duct obstruction. Gut 1998; 43: 680-683

[6] Feldman DR, Kulling DP, Kay CL et al. Magnetic resonance cholangiopancreatography: a novel approach to the evaluation of suspected pancreaticobiliary neoplasms. Ann Surg Oncol 1997; 4: 634-638

[7] Fulcher AS, Turner MA, Capps GW et al. Half-Fourier RARE MR cholangiopancreatography: experience in 300 subjects. Radiology 1998; 207: 21-32

[8] Rosch T, Meining A, Fruhmorgen $S$ et al. A prospective comparison of the diagnostic accuracy of ERCP, MRCP, CT, and EUS in biliary strictures. Gastrointest Endosc 2002; 55: 870-876

[9] Nishikawa T, Tsuyuguchi T, Sakai Y et al. Preoperative assessment of longitudinal extension of cholangiocarcinoma with peroral videocholangioscopy: a prospective study. Dig Endos 2014; 26: 450-457

[10] Itoi T, Sofuni A, Itokawa F et al. Peroral cholangioscopic diagnosis of biliary-tract diseases by using narrow-band imaging (with videos). Gastrointest Endosc 2007; 66: 730-736 